\title{
The centrality of event scale: A measure of integrating a trauma into one's identity and its relation to post-traumatic stress disorder symptoms
}

\author{
Dorthe Berntsen $^{\mathrm{a}, *}$, David C. Rubin ${ }^{\mathrm{b}}$ \\ ${ }^{a}$ Department of psychology, University of Aarhus, Jens Chr. Skous Vej 4, 8000 Aarhus C, Denmark \\ ${ }^{\mathrm{b}}$ Duke University, USA
}

Received 4 June 2004; received in revised form 29 December 2004; accepted 24 January 2005

\begin{abstract}
We introduce a new scale that measures how central an event is to a person's identity and life story. For the most stressful or traumatic event in a person's life, the full 20-item Centrality of Event Scale (CES) and the short 7-item scale are reliable ( $\alpha$ 's of .94 and .88, respectively) in a sample of 707 undergraduates. The scale correlates .38 with PTSD symptom severity and .23 with depression. The present findings are discussed in relation to previous work on individual differences related to PTSD symptoms. Possible connections between the CES and measures of maladaptive attributions and rumination are considered along with suggestions for future research.
\end{abstract}

(C) 2005 Elsevier Ltd. All rights reserved.

Keywords: Identity; Trauma; PTSD; Autobiographical memory; Individual differences

\section{Introduction}

Highly accessible and vivid personal memories help to give meaning and structure to our life narratives and help to anchor and stabilize our conceptions of ourselves (e.g., Baerger \& McAdams, 1999; Pillemer, 1998, 2003; Robinson, 1992; Robinson \& Taylor, 1998; Shum, 1998).

\footnotetext{
${ }^{*}$ Corresponding author.

E-mail address: dorthe@psy.au.dk (D. Berntsen).
} 
However, although generally overlooked, the outcome of such processes need not always be positive. If memories of traumas and other highly negative life events form reference points for the organization of less salient experiences in a person's life, the outcome is likely to be harmful to that person's mental health (Berntsen, Willert, \& Rubin, 2003). In such cases, a highly negative, unpredictable, and probably rare event will influence the attribution of meaning to other more mundane events as well as the generation of expectations for future events. Ruminations, unnecessary worries, and compulsive attempts at avoiding similar events in the future are likely outcomes. We present a new scale - the Centrality of Event Scale (CES) - to measure the extent to which a memory for a stressful event forms a reference point for personal identity and for the attribution of meaning to other experiences in a person's life. As we will show, the CES shows high internal consistency and substantial positive correlations with measures of PTSD symptoms. We clarify the theoretical rationale for the CES before reviewing previous studies on the salience and centrality of trauma memories in relation to PTSD symptoms.

\section{The centrality of traumatic memories}

Studies on subjective well-being indicate that traumatic and highly negative events are rare, compared to events perceived as positive or neutral (Diener \& Diener, 1996). Consistent with this view, most people seem to remember substantially more positive than negative events from their lives (for a review, see Walker, Skowronski, \& Thompson, 2003). A conventional life script (at least in our culture) contains more positive than negative events (Berntsen \& Rubin, 2004); the positive events are often about culturally expected role transitions, such as a graduation or a wedding. Such events help to keep the life story thematically and temporally coherent (Luborsky, 1993). In spite of such an overall pleasantness bias in autobiographical memory (Walker et al., 2003), memories for traumas and highly stressful events are in most cases very well remembered when compared to other autobiographical events (e.g., Berntsen, 2001; Porter \& Birt, 2001; Reviere \& Bakeman, 2001; Rubin, Feldman, \& Beckham, 2004). The literature on autobiographical memory and the availability heuristic suggests several possible functions for vivid and highly accessible personal memories. In most cases, the examples and analyses provided deal with possible functions of memories for relatively positive events, often culturally expected life events, such as a leaving home, or the birth of one's first child (e.g., Shum, 1998; Robinson, 1992). In the following, we argue that similar functions may be served by memories of traumas and other highly negative events. We delineate some possible maladaptive consequences and argue that they are related to symptoms associated with traumatization.

\section{Reference points for everyday inferences}

Memories for personally salient events may function as personal reference points for the attribution of meaning to other experiences as well as for the generation of expectations for the future. Such personal reference points can be seen as similar in many respects to what Pillemer (1998) labels as anchoring events and describes as "a lasting reminder of the way things are" (p. 74). He provides many examples showing that people use vivid personal memories to validate current beliefs and feeling, and to guide thoughts and behaviour.

This role of specific memories in personal judgements can be related to the availability heuristic described by Tversky and Kahneman (1973) — that is, we judge the frequency and probability of 
specific classes of events by the ease with which we are able to retrieve them. In most cases, this heuristic works well, because frequent events are generally also highly accessible in memory. However, following the availability heuristic, because trauma memories are generally highly accessible, a person with a memory for a trauma is likely to overestimate the general frequency of such events as well as the likelihood of being traumatized again in the future. One possible outcome may be unnecessary worries and precautions, such as avoiding certain classes of events because they are perceived as involving particular risks.

\section{Turning points in life stories}

Several scholars have described how salient personal memories form turning points or landmarks in the life story of the person (e.g., Baerger \& McAdams, 1999; Pillemer, 1998, 2003; Robinson, 1992; Robinson \& Taylor, 1998; Shum, 1998). Though the arguments and the examples they have provided usually have been about non-traumatic memories, we will extrapolate the findings to memories for traumas and other negative, stressful events. Pillemer (1998) offers an extensive account of possible functions of memories for momentous events, which he defines as personal memories referring to specific moments in the past characterized by "importance, definiteness and brevity" (p. 27) - a description that applies to many traumatic encounters and other vivid memories (Brown \& Kulik, 1977; Rubin \& Kozin, 1984). According to Pillemer (1998), such events are likely to function as turning points - that is, "a specific episode, or series of episodes, [that] appears to alter or redirect the ongoing flow of the life course" (p. 76). Following this definition, a turning point is a causal agent in the life story. It provides some explanations on the subsequent choices, acts and values of the person. It can be seen as the end of one chapter of the life story and the beginning of another (Pillemer, 1998).

Because a traumatic or highly stressful event often causes profound changes in a person's outlook (Janoff-Bulman, 1988), often stays highly accessible for years, and often comes to mind spontaneously in response to internal and external cues (Berntsen, 2001), it may be perceived as a major causal agent and thus as a highly salient turning point in the person's life. However, at the same time, having a trauma as a salient turning point in the life story may lead to oversimplifications. It may cause the person to focus on those aspects of his or her current life conditions that can be explained by reference to the trauma, and to ignore aspects that defy such causal attributions. It can therefore be seen as a way of optimizing the internal consistency of the life story at the expense of the multiplicity of meaning that normally characterizes our life narratives (Linde, 1993; Robinson, 1996).

\section{Components of personal identity}

According to theorists of autobiographical memory, the way we compose our life stories is closely related to the way we understand ourselves. For example, Fitzgerald (1988) described a life story as a "set of stories that defines who we are in narrative rather than declarative terms" (p. 269). Thus, if a trauma memory is seen as a central turning point in our life story it would also most likely be regarded as a central component of our personal identity.

Furthermore, having a traumatic event as central to personal identity is likely to mean that the trauma is seen as emblematic for the person's self and/or as a symbol for persistent themes in the person's life story. This in turn may pave the road for what Abramson and Seligman (1978) called internal, stable and global attributions: The trauma is seen as causally related to stable 
characteristics of the self that pertain across situations. This attributional style is positively related to depression (Peterson \& Seligman, 1984) and to PTSD symptoms (Greening, Stoppelbein, \& Docter, 2002). Brewin (2003) also discusses several ways in which a trauma may affect identity.

\section{Previous research relevant to the CES}

Clinical case studies and observations have indicated that traumatic memories are fragmented and lack narrative coherence as compared to other memories both with respect to the narrative coherence of the trauma memory when viewed in isolation and with respect to the integration of the trauma memory into the overall life story and self-schema of the person (see Reviere \& Bakeman, 2001, for a review). However, both claims have found little support when subjected to more systematic experimental examination (e.g., Berntsen et al., 2003; Gray \& Lombardo, 2001; Porter \& Birt, 2001; Reviere \& Bakeman, 2001; Rubin et al., 2004; but see Halligan, Michael, Clark, \& Ehlers, 2003). Because the CES is primarily concerned with the second part of the claim - that is, the integration of the trauma memory into the overall life story and self-schema of the person-our review of previous work will focus on this issue.

Byrne, Hyman, and Scott (2001) asked 77 undergraduates who reported having had a traumatic event in their past to rate on a 7-point scale how important this event was to their current understanding of themselves. Ratings of importance to self-understanding correlated positively with PTSD symptoms $(r=.35)$.

Berntsen et al. (2003) asked two questions included in the present CES (items 2 and 3 in the appendix) to 113 participants with traumatic events in their past. A reanalysis of Berntsen et al.'s data showed that the two questions were positively correlated $(r=.58)$. Participants who had a symptom profile corresponding to the diagnostic criteria for PTSD scored higher on both questions than participants without. Moreover, scores on "I feel the traumatic event has become part of my identity" were positively correlated with frequency of intrusive memories in both the PTSD and the non-PTSD symptom group ( $r \mathrm{~s}=.48$ and .38 , respectively).

Berntsen and Thomsen (in press) examined reactions associated with stressful events during World War II in a sample of 145 elderly Danes who had lived through the German occupation of Denmark from 1940 to 1945. They included three questions that are part of the CES (items 2, 3, and 6 in the appendix). These three questions were formulated to address the centrality of the German occupation to participants identity and life story and had a Cronbach's $\alpha$ of .77. A composite score for the three questions correlated positively with frequency of intrusive memories $(r=.37)$ and dreams $(r=.35)$ referring to the time of the occupation and with the extent to which participants agreed that the time of the war had left a scar on their soul $(r=.57)$.

Rubin and Berntsen (2004) asked 111 undergraduates five questions which are now in the CES (items 1, 2, 3, 6 and 10 in the appendix), as well as a standardized checklist for PTSD symptoms (Blanchard, Jones-Alexander, Buckley, \& Foneris, 1996; Weathers, Litz, Huska, \& Keane, 1994). Both series of questions were answered for one or more stressful events in the participants lives, and did not necessarily cover a traumatic event as defined by the diagnostic criteria for PTSD (American Psychiatric Association, 1994). To clarify whether participants has had an experience that satisfied the diagnostic criteria for a trauma, participants also filled in a trauma checklist generated from the DSM-IV (American Psychiatric Association, 1994) and Foa (1995). In addition, they answered the Beck depression inventory (BDI) (Beck, Ward, Mendelson, Mock, \& 
Erbaugh, 1961), a set of questions related to their life story and a set of questions related to their life script (Berntsen \& Rubin, 2004). The composite score for the five CES questions was reliable (Cronbach's $\alpha=.91$ ) and correlated positively with PTSD symptoms $(r=.45)$ as well as with depression $(r=.36)$. A positive relation between the composite score for the five CES questions and PTSD symptoms was found both among participants with and without events corresponding to the A1 and A2 criteria for traumas in the PTSD diagnosis (American Psychological Association, 1994).

In sum, individuals differ with respect to the extent to which a highly emotionally negative event has become central to their identity, life story and understanding of the world, and such individual differences seem to be critically related to PTSD symptomatology. Given these findings, we decided to develop a standardized scale measuring the extent to which a traumatic or stressful event forms a personal reference point for the attribution of meaning to other events, a salient turning point in the life story and a central component of a person's identity and selfunderstanding. We expect scores on this scale to be positively related to symptoms of PTSD and possibly related disorders.

\section{Method}

\section{Developing the CES}

In consultation with our colleagues, we generated a total of 23 scale items from our previous research and from the literature on the three interrelated theoretical themes in autobiographical memory and the availability heuristic, reviewed in the introduction. Three of the 23 items are not included in the final version of the CES represented in the appendix for reasons to be clarified later. Among the 20 items in the appendix, items 1, 2, 4, 9, 12, 13, 17 and 20 were generated to primarily address whether the event (or series of events) had become a reference point (or anchor point) for the generation of expectations and attribution of meaning to other events in the person's life. Items 3, 5, 6, 7, 8, and 19 in the appendix primarily addressed whether the event (or series of events) was perceived as a central component of personal identity. Items 10, 14, 15, 16, and 18 in the appendix addressed whether the event (or series of events) was regarded as a turning point in the life story. Five point Likert scales were chosen to be consistent with our preliminary work on the CES scale (e.g., Berntsen et al., 2003) as well as with the scale we used to measure PTSD symptoms (the PCL, see Blanchard et al., 1996).

\section{Participants}

The questionnaire was given to 707 undergraduates from four North American universities: Duke University $(n=224)$, North Carolina State University $(n=62)$, Wayne State University $(n=134)$, and University of North Dakota $(n=287)$. At Duke University, the average age was $18.73(\mathrm{SD}=.93) ; 54 \%$ female; $11 \%$ African American, 16\% Asian, 63\% Caucasian, 6\% Hispanic, $1 \%$ Native American, and 3\% other. At North Carolina State University, the average age was $19.75(\mathrm{SD}=4.01) ; 47 \%$ female; 15\% African American, 78\% Caucasian, and 7\% other. At Wayne State University, the average age was $20.50(\mathrm{SD}=5.13) ; 67 \%$ female; $41 \%$ African 
American, $5 \%$ Asian, $48 \%$ Caucasian, and $6 \%$ other. At the University of North Dakota, Grand Forks, the participants were drawn from a population with average age was $19.82(\mathrm{SD}=2.22)$; 67\% female; $1 \%$ African American, 1\% Asian, 94\% Caucasian, 1\% Hispanic, and 3\% Native American.

\section{Procedure}

All questionnaires were administered in groups and were included with other experimental material or screening scales. The Duke undergraduates also completed the posttraumatic stress disorder checklist (PCL; Blanchard et al., 1996; Weathers et al., 1994) and the BDI. Answers to the CES and PCL were based on participants' most stressful or traumatic event. The 23 CES items were presented on one sheet of paper. In addition to the 20 items in appendix, the following three questions were included: "I see many connections and similarities between this event and memories from other parts of my life" (presented as item number 4). I do not think that it was entirely a coincidence that this event happened to me (presented as item number 15). "If somebody made a two hour movie about my life, this event would be in the movie "(presented as item number 23).

On the reverse side of the same sheet of paper, two questions were reworded from the DSM IV diagnostic criteria for having a trauma and were intended to measure the A1 and A2 criteria for PTSD: (A1) "In the most stressful or traumatic event that you used to answer questions 1-23, did you experience, witness, or were you confronted with an event that involved actual or threatened death or serious injury, or threat to your physical integrity or that of others" (yes, no). (A2) "In the most stressful or traumatic event that you used to answer questions 1-23, did your response involve intense fear, helplessness, or horror" (yes, no). These two questions were preceded by a question addressing which emotion was the most dominant in the event, which is not analysed here.

\section{Results}

In order to ensure that all questions were measuring the same scale, we evaluated the degree to which scores on each question correlated with the sum of the scores on all other questions in the combined sample of all undergraduates and in each sample individually. The three questions (listed as number 4, 15, and 23 in the procedural section) had correlations with the sum of the other questions less than .52 in the combined sample and were also among the lowest correlations in the individual samples. Because they were also observed to be either vague (item 4) or to introduce slightly different themes than the concept tapped by the CES (e.g., fatalism for item 15, self-exposure for item 23), they were removed. The 20 remaining items (as presented in the appendix) had correlations between .55 and .72 (mean $=.64, \mathrm{SD}=.05$ ). The Cronbach's $\alpha$ of the remaining 20 questions was .94 for the combined sample and varied between .93 and .95 for the four universities.

Because the 20-item scale had such a high reliability, we constructed a shorter scale. The seven questions with the highest correlations with the sum of the other questions were chosen (items number $3,6,10,12,16,17$, and 18). The 7-item scale had a Cronbach's $\alpha=.88$ (range of $.87-.89$ 
for the four universities) and covers the range of key theoretical properties of the CES. The 20 and 7 item CES were correlated .96 (range of .96-.97 across the four universities). The 7-item version of the CES had a Cronbach's $\alpha=.92$ when tested in a new sample of 216 Duke undergraduates.

In order to ensure that we did not have more than one concept underlying the CES, we performed an iterated principle factor analyses with a varimax rotation. On the 7-item CES, only one eigenvalue was above 1.00 , with a value of 4.04. On the 20-item CES, 3 eigenvalues were greater than 1.00: 9.38, 1.41, and 1.02, with a smooth continuous drop for the remaining eigenvalues, making the clearest break in the Scree Plot between the first and second factor, again suggesting a one factor solution. When a rotated three factor solution was tried, the only interpretable pattern we could find was that two questions beginning with the phrase "I believe that people who haven't experienced this type of event" loaded highly on the third factor. Thus, we appear to have one underlying dimension. The following analyses are based on the 20-item version of the CES, unless otherwise specified.

We examined whether the magnitude of the CES was affected by whether the event was judged to involve actual or threatened death or serious injury (the A1 criterion of the DSM IV, American Psychiatric Association, 1994) or involve intense fear, helplessness, or horror (the A2 criterion), or both (the A criterion). Yes answers were given to the A1, A2, and both the A1 and A2 questions by $61 \%, 50 \%$, and $38 \%$ of the participants, respectively. The means for the CES for having or not having the A1 criterion were 2.98 versus $3.00(F(1,668)=0.12$, ns). The means for the CES for having or not having the A2 criterion were 3.07 versus $2.84(F(1,668)=10.65, p<.01)$. The means for the CES for having or not having the A criterion were 3.04 versus $2.95(F(1,705)=1.71$, ns). Comparing across universities, there were no differences in the level of the CES $(F(3,668)=0.08)$. For the three (one each for the A1, A2, and A criteria ) two-ways ANOVAs with CES score as the dependent variable and university population and having versus not having the criteria concerned as independent variables, there were no significant interactions (maximum $F(3,669)=1.97$, $p=0.12$ ). Thus, the CES is insensitive to whether or not people report having an event that matches the A1 diagnostic criteria for a trauma, but shows a significant difference for the A2 criteria (American Psychiatric Association, 1994).

The Duke undergraduates also completed the BDI and PCL, providing scores for depression, severity of PTSD symptoms overall, as well as the subscales for reliving (B symptoms), avoidance (C symptoms), and arousal (D symptoms). The correlation over the 224 Duke undergraduates between the 20-item CES and the PCL and BDI were $.38(p<.0001)$ and $.23(p<.01)$. For the 7item CES these correlations were $.37(p<.0001)$ and $.24(p<.001)$. The PCL and BDI correlated $.43(p<.0001)$.

Table 1 provides a more complete description by providing the $\mathrm{B}, \mathrm{C}$, and $\mathrm{D}$ symptoms separately from the PCL and also including the A1, A2, and A symptoms. As can be seen in Table 1 , there are moderate correlations among the CES, BDI, and PCL subscales, as well as among the A criteria for PTSD, but these two clusters of variables do not correlate with each other.

As a further attempt at examining the usefulness of the CES in relation to PTSD symptoms, we predicted the total PCL score with CES, BDI, and the A1 and A2 criteria, with independent variables entering at the $p<.05$ level. The resulting equation using beta weights was: $\mathrm{PCL}=.36$ $\mathrm{BDI}+.30 \mathrm{CES}, R^{2}=.27$. If we instead try to predict depression symptoms, we get $\mathrm{BDI}=.40$ $\mathrm{PCL}+.11 \mathrm{~A} 2, R^{2}=.17$ with the CES not entering. Thus, depression was predicted by the PCL and by a sense of horror or helplessness at the time of the event, but not by the CES. 
Table 1

Correlations among tests for duke undergraduates

\begin{tabular}{|c|c|c|c|c|c|c|c|}
\hline & CES & BDI & B & $\mathrm{C}$ & $\mathrm{D}$ & A1 & A2 \\
\hline BDI & .23 & & & & & & \\
\hline B & .32 & .30 & & & & & \\
\hline C & .35 & .43 & .62 & & & & \\
\hline D & .28 & .34 & .62 & .65 & & & \\
\hline A1 & -.13 & -.02 & .03 & -.03 & -.03 & & \\
\hline A2 & -.02 & .14 & .05 & .07 & .07 & .27 & \\
\hline A & -.06 & -.01 & .06 & .03 & -.00 & .76 & .62 \\
\hline
\end{tabular}

Note: B refers to the B-symptoms of PTSD which involve reliving, C-symptoms involve avoidance, and D-symptoms involve arousal and hypervigilance, A1 refers to the traumatic nature of the event itself, A2 to the reaction to the event, and A requires both.

Table 2

Scores for duke undergraduates with high and low PCL scores

\begin{tabular}{lccc}
\hline & PCL $>44$ & PCL $\leqslant 44$ & $F(1,222) p$ \\
Mean (SD) & Mean (SD) & \\
\hline Number & 37 & 187 & \\
PCL & $52.65(6.92)$ & $28.22(7.12)$ & $18.69<.0001$ \\
BDI & $11.23(7.00)$ & $6.42(5.76)$ & $21.23<.0001$ \\
CES & $3.56(.80)$ & $2.84(.89)$ & $.16=.69$ \\
A1 $(\%)$ & $52.68(48.51)$ & $49.19(49.04)$ & $.03=.87$ \\
A2 $\%)$ & $58.93(48.83)$ & $60.32(47.99)$ & $.35=.55$ \\
A $(\%)$ & $40.81(47.85)$ & $35.79(47.02)$ & \\
\hline
\end{tabular}

One concern is that in the results reported, a few participants whose symptoms were severe enough to probably have PTSD if diagnosed by a clinician were swamped by the many participants without symptoms. Fortunately, the PCL test we administered agrees extremely well with a full clinical diagnosis. Blanchard et al. (1996) correctly classified 17 out of 18 PTSD patients using a score of above 44 as an indicator of PTSD. We therefore divided our sample using this cutoff. The results, which are in Table 2, show that participants with high levels of PTSD symptoms differ from those with lower levels on the BDI and CES, but not on A symptoms.

\section{Discussion}

We have introduced a new scale that measures the extent to which a memory for a stressful event forms a reference point for personal identity and for the attribution of meaning to other experiences in a person's life. For the most stressful or traumatic event in a person's life, the full 20 -item CES and the short 7-item scale were reliable ( $\alpha^{6}$ s of .94 and .88, respectively) in a sample of 707 undergraduates. When retested in a sample of 216 Duke undergraduates, the 7-item scale 
showed a Cronbachs $\alpha$ of .92 . The 20-item scale correlated .38 with PTSD symptom severity and .23 with depression, but not with whether a person reported an event that matched the current diagnostic criteria for a trauma.

The findings indicate that the mechanisms measured by the CES are important for the understanding of individual differences associated with PTSD symptoms. Here we tested a general undergraduate population with and without trauma and with and without symptoms of PTSD to obtain a general sense of the reliability of the scale and to test the generality of the concept of centrality of stressful or traumatic events to one's identity. We showed that participants with a PCL-score above the critical level indicating PTSD had markedly higher scores on the CES compared to others, independent of whether they had a trauma as defined by the current diagnosis of PTSD (American Psychiatric Association, 1994).

The finding that the PCL-score was unrelated to whether the person had an event that satisfied the A-criteria for a trauma in the DSM-IV (American Psychiatric Association, 1994) disagrees with results from meta-analyses in this area, showing that perceived life danger and peritraumatic emotion are significantly related to severity of PTSD symptoms (Brewin, Andrews, \& Valentine, 2000; Ozer, Best, Lipsey, \& Weiss, 2003). The explanation may be that most of the previous studies reviewed in the meta-analyses were based on traumatic events with a high likelihood of life danger (combat experiences, disasters, accidents, and interpersonal violence) and (with respect to Ozer et al., 2003) that measures of peritraumatic emotion included measures of guilt and shame in addition to fear, helplessness and horror and thus did not correspond strictly to the A2 trauma criterion. In contrast, participants in the present study were asked only about fear, horror and helplessness consistent with the A2 trauma criterion, and the present study involved undergraduates with a wider range of stressful events. The latter is important because previous research has shown that the effects of different risk factors for PTSD (such as trauma severity) vary across different categories of traumatic events (Brewin et al., 2000). Because many of our participants based their answers on stressful events that did not seem to satisfy the A1 and A2 criterion for a trauma, future research should examine the relation between the CES and PTSD symptoms in clinical populations who fulfill the diagnostic criteria for traumas (for a discussions see Brewin, 2003; McNally, 2003). The CES score need not increase linearly with severity of PTSD symptoms. Because of this and because of the restricted range of PTSD symptom severity within a clinical population, the CES may not show correlations with symptom severity within a population of PTSD patients that are similar to the correlations found in the present study.

Although we have advocated the view that trauma memories in PTSD are central to a person's identity and life story and easily accessed in everyday inferences and heuristics, this does not imply that dissociative processes play no role in PTSD. Having a traumatic memory as highly accessible and central in the overall cognitive organization of autobiographical knowledge is likely to lead to vivid, intrusive memories and thereby generate a need by the person for distancing him- or herself from the phenomenologically painful reliving of the trauma and its associated emotions. It may thus lead to the activation of dissociative coping techniques, such as observer perspective in remembering (see Berntsen et al., 2003, McIsaac \& Eich, 2004). This assumption is supported by our earlier work (Berntsen et al., 2003) in which we showed that participants with traumas and a set of symptoms indicating PTSD showed relatively high positive correlations between memory characteristics taken to indicate dissociation, on the one hand, and the severity of the traumatic event and vividness of the traumatic recollection, on the other. Such pattern was not observed 
among participants without symptoms indicating PTSD. At the same time, participants with a PTSD symptom profile agreed more with the statements that the trauma was central to their identity and they perceived more connections and similarities between the trauma and current experiences than participants without a PTSD symptom profile, consistent with the present theoretical framework.

Other theories also emphasize the cognitive organization of the trauma memory as a mediator of PTSD symptoms. There is a long tradition for viewing posttraumatic stress reactions, such as intrusive memories, as reflecting an inability to process and integrate the trauma with knowledge of the self and world (e.g., Horowitz, 1986, pp. 85-110). Our account differs from such accounts by arguing that the trauma memory is not poorly integrated into the person's life story and identity. In fact, the opposite is the case, according to our claims: The trauma has become too central to the cognitive organization of the life story and identity of the person.

In order to enlarge our understanding of the underlying psychological operations measured by the CES, an important question for future research is the relation between the CES and other psychological scales. For example, future research should examine possible relations between the CES and other measures of maladaptive cognitive processing styles in response to stressful life events, such as the attributional style questionnaire (ASQ) (Peterson et al., 1982). As pointed out by Peterson and Seligman (1984) the notion of attribution is a hypothetical construct that is not exhaustively defined or explained by a single underlying operation. We believe some of the operations that we have described as part of our theoretical rationale for the CES may be related to the maladaptive attributions measured by the ASQ. The existence of a positive correlation between scores on the CES and measures of depression can be seen as pointing in this direction. Also, certain personality factors, such as neuroticism (McCrae \& Costa, 1999) may be related to operations measured by the CES. Likewise, it is reasonable to expect that ruminating about negative events (Nolen-Hoeksema \& Morrow, 1991) is positively associated with the centrality of such events to the identity and life story of the person possibly leading to a positive relation between scores on the CES and measures of rumination. In addition, future research should examine whether scores on the CES are positively related to other disorders than PTSD and depression. For example, if the event being rated is changed from the general "most stressful or traumatic event in your life" to something more specific, the questionnaire might be adapted for other anxiety disorders.

In sum, it is well-known that the development of PTSD in response to stressful events has large individual differences (e.g., Brewin et al., 2000; McNally, Bryant, \& Ehlers, 2003; Ozer et al., 2003). The CES and the findings introduced here add to this literature by demonstrating that a certain way of thinking and narrating about stressful events is positively correlated with PTSD symptoms.

\section{Acknowledgment}

We wish to thank Adriel Boals, F. Richard Ferraro, Joseph Fitzgerald, and Amy Wenzel for their help in providing the non-Duke samples, Adriel Boals, Daniel Greenberg, Tim Strauman, and Jennifer Talarico for comments, and National Institute of Mental Health Grant no. R01 MH066079 for funding. 


\section{Appendix. The centrality of events scale ${ }^{1}$}

Please think back upon the most stressful or traumatic event in your life and answer the following questions in an honest and sincere way, by circling a number from 1 to 5 .

1. This event has become a reference point for the way I understand new experiences.

2. I automatically see connections and similarities between this event and experiences in my present life.

* 3. I feel that this event has become part of my identity.

4. This event can be seen as a symbol or mark of important themes in my life.

5. This event is making my life different from the life of most other people.

* 6. This event has become a reference point for the way I understand myself and the world.

7. I believe that people who haven't experienced this type of event think differently than I do.

8. This event tells a lot about who I am.

9. I often see connections and similarities between this event and my current relationships with other people.

*10. I feel that this event has become a central part of my life story.

11. I believe that people who haven't experienced this type of event, have a different way of looking upon themselves than I have.

*12. This event has colored the way I think and feel about other experiences.

13. This event has become a reference point for the way I look upon my future.

14. If I were to weave a carpet of my life, this event would be in the middle with threads going out to many other experiences.

15. My life story can be divided into two main chapters: one is before and one is after this event happened.

*16. This event permanently changed my life. totally disagree 12345 totally agree totally disagree 12345 totally agree

totally disagree 12345 totally agree totally disagree 12345 totally agree totally disagree 12345 totally agree totally disagree 12345 totally agree totally disagree 12345 totally agree totally disagree 12345 totally agree totally disagree 12345 totally agree

totally disagree 12345 totally agree totally disagree 12345 totally agree totally disagree 12345 totally agree totally disagree 12345 totally agree totally disagree 12345 totally agree totally disagree 12345 totally agree totally disagree 12345 totally agree

\footnotetext{
${ }^{1}$ Items in the 7-item version are marked with an asterix. The copyright for the scales is held by the authors (C) 2005, Berntsen \& Rubin). Permission is given to use the scales for research purposes.
} 
*17. I often think about the effects this event will have on my future.

*18. This event was a turning point in my life. 19. If this event had not happened to me, I would be a different person today.

20. When I reflect upon my future, I often think back to this event. totally disagree 12345 totally agree

totally disagree 12345 totally agree totally disagree 12345 totally agree

totally disagree 12345 totally agree

\section{References}

Abramson, L. Y., \& Seligman, M. E. P. (1978). Learned helplessness in humans: Critique and reformulation. Journal of Abnormal Psychology, 87, 49-74.

American Psychiatric Association. (1994). Diagnostic and statistic manual of mental disorders (4th ed.). Washington, DC: American Psychiatric Association.

Baerger, D. R., \& McAdams, D. P. (1999). Life story coherence and its relation to psychological well-being. Narrative Inquiry, 9, 69-96.

Beck, A. T., Ward, C. H., Mendelson, M., Mock, J., \& Erbaugh, J. (1961). An inventory for measuring depression. Archives of General Psychiatry, 4, 561-571.

Berntsen, D. (2001). Involuntary memories of emotional events. Do memories of traumas and extremely happy events differ? Applied Cognitive Psychology, 15, 135-158.

Berntsen, D., \& Rubin, D. C. (2004). Cultural life scripts structure recall from autobiographical memory. Memory \& Cognition, 32, 427-442.

Berntsen, D., \& Thomsen, D. K. (in press). Personal memories for remote historical events. Accuracy and clarity of flashbulb memories related to World War II.

Berntsen, D., Willert, M., \& Rubin, D. C. (2003). Splintered memories or vivid landmarks? Qualities and organization of traumatic memories with and without PTSD. Applied Cognitive Psychology, 17, 675-693.

Blanchard, E. B., Jones-Alexander, J., Buckley, T. C., \& Foneris, C. A. (1996). Psychometric properties of the PTSD checklist (PCL). Behaviour, Research and Therapy, 34, 669-673.

Brewin, C. R. (2003). Posttraumatic stress disorder. Malady or myth. New Haven: Yale University Press.

Brewin, C. R., Andrew, B., \& Valentine, J. D. (2000). Meta-analysis of risk factors for posttraumatic stress disorder in trauma-exposed adults. Journal of Consulting and Clinical Psychology, 68, 748-766.

Brown, R., \& Kulik, J. (1977). Flashbulb memories. Cognition, 5, 73-99.

Byrne, C. A., Hyman, I. E., \& Scott, K. L. (2001). Comparison of memories for traumatic events and other experiences. Applied Cognitive Psychology, 15, 119-133.

Diener, E., \& Diener, C. (1996). Most people are happy. Psychological Science, 7, 181-185.

Fitzgerald, J. M. (1988). Vivid memories and the reminiscence phenomenon: The role of a self narrative. Human Development, 31, 261-273.

Foa, E. B. (1995). Posttraumatic stress diagnostic scale (PDS). Minneapolis: National Computer Systems.

Gray, M. J., \& Lombardo, T. W. (2001). Complexity of trauma narrratives as an index of fragmented memory in PTSD: A critical analysis. Applied Cognitive Psychology, 15, 171-186.

Greening, L., Stoppelbein, L., \& Docter, R. (2002). The mediating effects of attributional style and event-specific attributions on postdisaster adjustment. Cognitive Therapy and Research, 26, 261-274.

Halligan, S. L., Michael, T., Clark, D. M., \& Ehlers, A. (2003). Posttraumatic stress disorder following assault: The role of cognitive processing, trauma memory, and appraisals. Journal of Consulting and Clinical Psychology, 71, 419-431.

Horowitz, M. J. (1986). Stress response syndromes (2nd ed.). Northvale, NJ: Jason Aronson.

Janoff-Bulman, R. (1988). Assumptive worlds and the stress of traumatic events: Applications of the schema construct. Social Cognition, 7, 113-136.

Linde, C. (1993). Life stories. The creation of coherence. New York: Oxford University Press. 
Luborsky, M. R. (1993). The romance with personal meaning in gerontology: Cultural aspects of life themes. The Gerontologist, 33, 445-452.

McCrae, R. R., \& Costa, P. T. (1999). A five factor theory of personality. In L. A. Pervin, \& O. P. John (Eds.), Handbook of personality, (2nd ed.) (pp. 139-153). New York: Guilford Press.

McIsaac, H. K., \& Eich, E. (2004). Vantage point in traumatic memory. Psychological Science, 15, 248-253.

McNally, R. J. (2003). Progress and controversy in the study of posttraumatic stress disorder. Annual Review of Psychology, 54, 229-252.

McNally, R. J., Bryant, R. A., \& Ehlers, A. (2003). Does early psychological intervention promote recovery from posttraumatic stress? Psychological Science in the Public Interest, 4, 45-79.

Nolen-Hoeksema, S., \& Morrow, J. (1991). A propspective study of depression and post-traumatic symptoms after a natural disaster: The 1989 Loma Prieta Earthquake. Journal of Personality and Social Psychology, 61, 115-121.

Ozer, E. J., Best, S. R., Lipsey, T. L., \& Weiss, D. S. (2003). Predictors of posttraumatic stress disorder and symptoms in adults: A meta-analysis. Psychological Bulletin, 129, 52-73.

Peterson, C., \& Seligman, M. E. (1984). Causal explanations as a risk factor for depression: Theory and evidence. Psychological Review, 91, 347-374.

Peterson, C., Semmel, A., von Baeyer, C., Abramson, L. Y., Metalsky, G. I., \& Seligman, M. E. P. (1982). The attributional style questionnaire. Cognitive Therapy and Research, 6, 287-299.

Pillemer, D. B. (1998). Momentous events, vivid memories. Cambridge: Harvard University Press.

Pillemer, D. B. (2003). Directive functions or autobiographical memory: The guiding power of the specific episode. Memory, 11, 193-202.

Porter, S., \& Birt, A. R. (2001). Is traumatic memory special? A comparison of traumatic memory characteristics with memories for other life experiences. Applied Cognitive Psychology, 15, 101-117.

Reviere, S. L., \& Bakeman, R. (2001). The effects of early trauma on autobiographical memory and schematic selfrepresentation. Applied Cognitive Psychology, 15, 89-100.

Robinson, J. A. (1992). First experiences: Contexts and functions in personal histories. In M. A. Conway, D. C. Rubin, H. Spinnler, \& W. Wagenaar (Eds.), Theoretical perspectives on autobiographical memory (pp. 223-239). Utrecht: Kluwer Academic Publishers.

Robinson, J. A. (1996). Perspective, meaning and remembering. In D. C. Rubin (Ed.), Remembering our past: Studies in autobiographical memory (pp. 199-217). Cambridge: Cambridge University Press.

Robinson, J. A., \& Taylor, L. R. (1998). Autobiographical memory and self-narratives: A tale of two stories. In C. P. Thompson, D. J. Herrmann, D. Bruce, J. D. Read, D. G. Payne, \& M. P. Toglia (Eds.), Autobiographical memory: Theoretical and applied perspectives (pp. 125-143). Mahwah, NJ: Lawrence Erlbaum Associates.

Rubin, D.C. \& Berntsen, D. (2004). Remembering the road less traveled makes a difference: Divergence from a life script correlates with psychological distress. Poster presented at the 10th Cognitive Aging Congress, Atlanta, April $1-4$.

Rubin, D. C., Feldman, M. E., \& Beckham, J. C. (2004). Reliving, emotions and fragmentation in the autobiographical memories of veterans diagnosed with PTSD. Applied Cognitive Psychology, 18, 17-35.

Rubin, D. C., \& Kozin, M. (1984). Vivid memories. Cognition, 16, 81-95.

Shum, M. S. (1998). The role of temporal landmarks in the autobiographical memory processes. Psychological Bulletin, $124,423-442$.

Tversky, A., \& Kahneman, D. (1973). Availability: A heuristic for judging frequency and probability. Cognitive Psychology, 5, 207-232.

Walker, R. W., Skowronski, J. J., \& Thompson, C. P. (2003). Life is pleasant-and memory helps to keep it that way. Review of General Psychology, 7, 203-210.

Weathers, F. W., Litz, B. T., Huska, J. A., \& Keane, T. M. (1994). The PTSD checklist (PCL). Unpublished scale available from the National Center for PTSD. 